\title{
Causal inference in epidemiologic researches: are we making the right decision?
}

\begin{abstract}
Often scientific researchers from different disciplines concluded the researches studies by stating that they found causality between exposures or cause and disease without making sure all the fundamental criteria were effectively met and verified. Should we continue undermining the quality of our research by not stating appropriately the relationship we found from the studies we conducted diligently and with respect to our scientific credibility?
\end{abstract}

Volume 4 Issue 2 - 2016

\author{
Koffitse Atchon \\ Department of Community, Rush University Medical Center, \\ USA \\ Correspondence: Koffitse Atchon, Adjunct Faculty, Rush \\ University Medical Center, Department of Community, Systems \\ and Mental Health Nursing, 600 S Paulina St., \#I060A, Chicago, \\ IL, USA, Tel I (3I2) 9425899, Email chkatchon@gmail.com
}

Received: January 26, 2016 | Published: February 02, 2016

\section{Editorial}

The causal inference is related to a co variation of the cause and effect. According to the Psychological dictionary defined causal inference as a manner of reasoning which permits an individual to see causal relationships in events and infer associations between and among them.

Most researches studies in the health, social and behavioral sciences may not be concerned about association but with causal relationship in the nature. Solving causal relationship systematically requires certain criteria that emphasize in the rigor of epidemiologic and statistical literature and education.

Sir Austin Bradford Hill also known as Bradford Hill (1897-1991) was an outstanding pioneer in medical statistics and epidemiology, who put forwards in 1965 an enormous influence on attempts to separate causal from non-causal explanations of observed associations. $^{2}$

As general rule, one must make sure the following criteria called The Bradford Hill causality criteria or guidelines ${ }^{3}$ are stated as follows:

a. Experimental evidence: experiments make a causal inference more plausible;

b. Temporality: the cause must precede the effect(start with individuals with disease and determine the disease onset is prior to the exposure);

c. The strength of association: we should make sure that the higher the odds ratios or the relative risks;

d. Dose response relationship (graded pattern): greater amounts of exposure should result in greater amount of harm;

e. Biological plausibility: there must be a rational and theoretical basis for the finding;

f. Consistency: multiple studies showing the same finding increases the credibility of the finding. In addition, the less important guidelines are as follows:

i. (Specificity (the effect has only one cause); ii. Coherence (cause-effect relationship doesn't conflict with what is known and there are no other competing hypotheses);

iii. Analogy (a commonly accepted phenomenon in one area can be applied to another area).

In contrast, the failure to satisfy any or even most of these guidelines does not necessary constitutes evidence that the association is not causal. ${ }^{4}$

However, we read in many publications that the researchers implied causality without ensuring and confirming that the criteria of HillBradford are met before assuming the causality between the first which proceeded and the second event (exposure or cause and disease). The lack of inadequate decisional findings made Bradford Hill to argue that none of these nine viewpoints can bring indispensable evidence for or against a cause-and-effect hypothesis and none can be required as a sine qua non. What they can do, with greater or less strength, is to help answer the fundamental question - is there any other way of explaining the set of facts before us, is there any other answer equally, or more, likely than cause and effect., ${ }^{5,6}$ This is a great reminder for researcher to make sure the Hill's criteria are met when they conclude their decision regarding the causal inferences, and preserve the quality of the researches findings.

The importance of considering the Bradford Hill's guidelines is crucial while making causal inference and decision in the relationship of consecutive events. The process of translating epidemiologic research findings or scientific evidences into health policies should involve careful considerations of issues such as bias and confounding as well as the ability to interpret the results of researches. Causal inferences may also have substantial economic implications for the community and those who might be held accountable for the exposure.

\section{Acknowledgements}

None.

\section{Conflict of interest}

The author declares no conflict of interest. 


\section{References}

1. Chalmers I. Fisher and Bradford Hill: theory and pragmatism? Int $J$ Epidemiol. 2003;32(6):922-924.

2. Hofler M. The Bradford Hill considerations on causality: a counterfactual perspective. Emerg Themes Epidemiol. 2005;2:11.
3. Szklo M, Nieto FJ. Epidemiology beyond the Basics. 2007.

4. Gordis L. Epidemiology. USA: Elsevier Sanders; 2004.

5. Hill AB. The environment and disease: association or causation? Proc $R$ Soc Med. 1965;58(5):205-300.

6. Pam. What is causal inference? Psychological Dictionary. 2013. 